\title{
La escrófula en el siglo XIX
}

\author{
Ignacio Duarte
}

\section{Scrofula in the $19^{\text {th }}$ century}

In the $19^{\text {th }}$ century scrofula or scrofulous adenitis was a frequent condition estimated by the finding of swollen cervical lymph nodes or scars, occurring in both sexes at all epochs of life, mainly in children. It was thought that it principally affected people with an inherited phlegmatic constitution that involved a scrofulous disposition or "diathesis". The disease would be triggered by environmental agents, bad habits or excesses in style of life. Besides injuring cervical lymph nodes, in some cases scrofula could compromise other groups of lymph nodes, bones, joints, lungs or other viscera. In some of its clinical presentations the disease could be healed while others were often lethal disorders. The finding of multiorgan compromise, caseation and "tuberculization" of the lesions originated discussion whether scrofula and tuberculosis were one or two different diseases and if they affected subjects with a common diathesis or people with a distinct scrofulous or tuberculous diathesis. Along the $19^{\text {th }}$ century, before the discovery of Koch's bacillus, the notion of contagion as a cause of scrofula and tuberculosis was not predominant in Europe.

Key words: Scrofula, tuberculosis, lymphadenitis, diathesis.

Palabras clave: Escrófula, tuberculosis, linfoadenitis, diátesis.

$\mathrm{E}$ 1 abultamiento crónico de los linfonodos cervicales recibió durante varios siglos el nombre de escrófula, término que aludía al aspecto porcinoide del cuello de los pacientes. En la medicina hipocrática se la atribuía a acumulación anormal de flema o pituita en los ganglios. Desde la antigüedad la escrófula atrajo el interés de los médicos por su evolución local que podía variar desde la remisión completa, la reaparición, la fistulización y cicatrización deformante ${ }^{1}$. Por mucho tiempo se creyó que ciertos reyes podían curarla milagrosamente ${ }^{2}$. A principios del medioevo se decía que el mal podía además comprometer otras partes del cuerpo, lo que se aceptó en los siglos XVI al XVIII por la observación macroscópica post mortem. Se describió la tisis escrofulosa, caracterizada por una tumefacción ganglionar cervical, con compromiso pulmonar y consunción, que se interpretaba como consecuencia de una acrimonia del componente líquido de la sangre, el cual se coagulaba en órganos esponjosos. El color y consistencia de las lesiones se comparó con el del queso o requesón y se reconoció que esta forma compleja de la enfermedad podía tener una evolución mortal ${ }^{1}$.

A fines del siglo XVIII y durante el siglo XIX se publicó un gran número de comunicaciones sobre la escrofulosis, posiblemente motivadas por la frecuencia con que los autores decían enfrentarse a la enfermedad y por las diferentes concepciones que se habían formado acerca ella ${ }^{3}$.

El objeto de esta publicación fue indagar acerca de las ideas que se planteaban en escritos europeos sobre la naturaleza, causas y variedades de la escrófula durante el siglo XIX, antes que Robert Koch descubriera el bacilo tuberculoso, en 1882.

\section{Aspectos clínicos}

En el siglo XIX se describía la característica aparición de tumoraciones nodulares del cuello, indolentes sin rubor ni calor al principio, pero que más tarde podían sufrir inflamación aguda con fiebre, afectación de la piel y del tejido celular. Luego los nódulos podían supurar y abrirse a la piel (Figura 1). Solían curar con lentitud y dificultad hacia la cicatrización: esto inducía la idea de que se había curado la afección. Sin embargo, esa sucesión podía repetirse con la consecuente deformación cicatricial de la zona y el temor de que persistiera un compromiso oculto.

Se consignaba que las escrófulas afectaban a personas de ambos sexos en todas las épocas de la vida. Durante la infancia los ganglios del cuello y los de la base de la mandíbula se comprometían con frecuencia; en la pubertad, principalmente los ganglios torácicos y el pulmón. En la infancia y después de la pubertad se afectaba de preferencia el mesenterio ${ }^{4}$.

En algunos casos el compromiso ganglionar podía acompañarse de lesiones cutáneas, oculares y catarros de mucosas. Podía también afectar directamente huesos, articulaciones y vísceras, evolucionando inclusive a una "caquexia escrofulosa"4. "En una palabra, ella no respeta ningún tejido y no se limita a atacar al sistema linfático como se ha creído: es una enfermedad de toda la economía".5.
Pontificia Universidad Católica de Chile.

Facultad de Medicina.

Programa de Estudios Médicos

Humanísticos.

Recibido: 29 de diciembre de 2016

Correspondencia a:

Ignacio Duarte García de Cortázar ignacioduarte.gc@gmail.com 
Figura 1. Paciente escrofuloso. Bramwell, Byrom. Edinburgh: Constable 1893. Atlas of Clinical Medicine. Fuente: National Library of Medicine, National Institutes of Health. Imagen de dominio público.

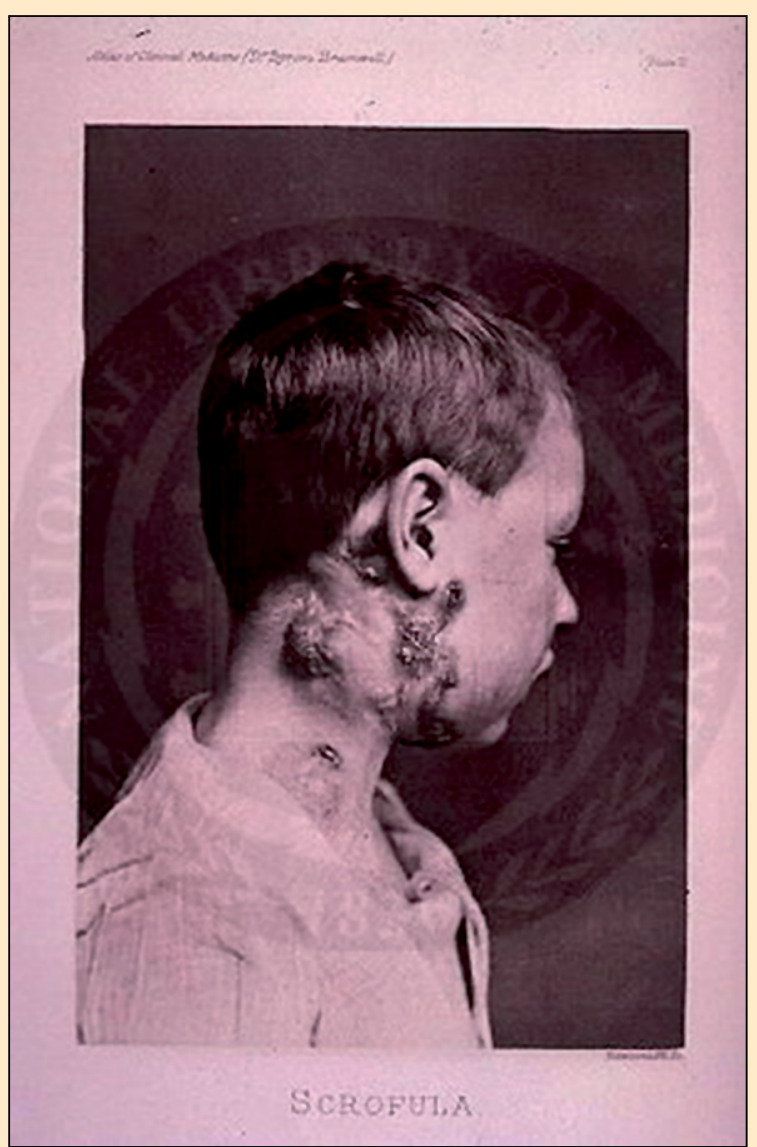

\section{Naturaleza de la enfermedad}

En el siglo XIX surgieron variados puntos de vista y discusiones acerca de la naturaleza de la escrofulosis y su relación con la enfermedad llamada tisis pulmonar, consunción o tuberculosis.

Algunos autores postulaban la existencia de dos entidades diferentes: una afección escrofulosa esencial y una afección tuberculosa esencial, aunque se reconocía que había una frecuente coincidencia de estas dos enfermedades en un mismo individuo ${ }^{6}$. Entre los argumentos que apoyaban esa posición se mencionaba que la tuberculosis era común en la adolescencia y juventud, su producto mórbido era siempre el mismo en cualquier lugar del cuerpo, la caquexia era extrema (el cuerpo se "secaba"), y su pronóstico era más grave. Por su parte, la escrófula era más frecuente en la infancia, se manifestaba por gran número y variedad de lesiones diversas, como abscesos, catarros, caries óseas y tubérculos; su evolución crónica era más lenta, la caquexia era "húmeda" por la presencia de lesiones edematosas, y no llegaba al marasmo ${ }^{7}$. Villemin afirmaba en 1868 que las afecciones escrofulosas tenían en común con la tuberculosis solo la consistencia caseosa de ciertas adenitis; este aspecto podía observarse también en otras adenitis como las de la sífilis, el muermo y la fiebre tifoidea. Sin embargo, la diferencia decisiva estribaba en que la escrofulosis era provocada por factores muy diversos y banales; al contrario, la causa de la tuberculosis era, según sus experimentos, una sola: un agente inoculable. Concluía que la tuberculosis era una unidad tanto en sus efectos como en su naturaleza esencial ${ }^{8}$.

Otros autores consideraban que ambas afecciones eran esencialmente la misma, siendo la tuberculosis el origen constitucional de la escrófula y la consunción 9 . En este sentido, se proponía que la escrofulosis correspondía a las manifestaciones externas y la tuberculosis más frecuentemente a las alteraciones internas ${ }^{10}$, designándose incluso a la tuberculosis como "la escrófula del pulmón" 11 .

Una idea bastante extendida sostenía que la condición fundamental de la escrófula era una constitución particular o "diátesis escrofulosa". Generalmente se consideraba congénita, transmitida por uno o ambos padres tuberculosos, escrofulosos o de mala salud. Se mantenía latente en el individuo hasta que obraban causas externas que desencadenarían la enfermedad. También se estimaba posible que esas mismas causas en algunos casos podían inducir la diátesis en forma adquirida ${ }^{12}$.

Se llegaron a describir dos tipos de constitución escrofulosa, atendiendo a manifestaciones físicas y conductuales de los individuos. El más frecuentemente citado era el tipo flemático o linfático: semblante imperturbable, labios gruesos, nariz ancha, piel gruesa y terrosa, pelo negro grueso, cuello ancho, huesos pesados, movimientos torpes, figura desgarbada, tardo de comprensión y con emociones débiles. Menos mencionado era el tipo sanguíneo o angélico: cara oval, piel clara, ojos azules grandes, pestañas largas, huesos largos y delgados, poca grasa corporal, mente precoz, modales nerviosos, bellos $y$ gráciles en la niñez ${ }^{13,14}$. Otros autores relacionaron la constitución escrofulosa con un predominio del humor bilis negra o melancolía ${ }^{15}$.

Para algunos las tumefacciones externas, catarros mucosos, úlceras cutáneas - a las que cabría agregar conjuntivitis, blefaritis, otitis, eczema, impétigo - eran meras consecuencias de la constitución especial del paciente ${ }^{16}$. Según Phillips, la prueba de que el individuo tenía la constitución escrofulosa era la tumefacción de ganglios linfáticos subcutáneos caracterizada por el depósito del producto denominado materia escrofulosa ${ }^{17}$.

Frente a la consideración de la escrófula y la tuberculosis como manifestaciones de diátesis diferentes, se propuso la alternativa de que ambas enfermedades se originaban en una diátesis única, llamada "escrofulismo". Esta idea se basaba principalmente en la distribución corporal, caracteres de las lesiones y frecuente coexistencia en un mismo individuo. El escrofulismo, fuera hereditario $o$ adquirido, constituiría una primera fase que a continua- 
ción podría manifestarse por accidentes secundarios en la piel ${ }^{18}$, mucosas (catarros), adenitis, caries y necrosis óseas. La tuberculosis declarada sería la fase terciaria. La segunda y tercera fase podrían presentarse sucesivamente o cada una en forma independiente ${ }^{12}$.

\section{Frecuencia}

Resulta difícil precisar la frecuencia de la escrófula debido a la variedad de afecciones que podían incluirse bajo tal diagnóstico. Se estimaba que en Inglaterra apenas alguna familia podía declarar que no la había afectado ${ }^{11}$. En los registros de sus pacientes, un cirujano informaba que figuraban 1.200 casos de escrofulosos ${ }^{19}$. Phillips reunió cifras de varias investigaciones y obtuvo las siguientes frecuencias en distintos grupos de personas examinadas: con cicatrices de escrófula 1 a $2 \%$; con ganglios abultados a la inspección, menos de 3\%. En 8\% de individuos pobres mayores de 16 años se encontraron ganglios abultados a la palpación ${ }^{17}$. Dubos refiere que casi todos los niños de un hospicio de Kent y 53\% de los de un orfanato de Berlín eran escrofulosos, y que en el ejército francés alrededor de $1,7 \%$ de los soldados sufría la enfermedad ${ }^{20}$.

\section{Causas}

Se proponía gran variedad de causas desencadenantes de la aparición de las escrófulas: habitar en grandes ciudades, en lugares húmedos y pantanosos, en viviendas hacinadas, falta de abrigo, ambientes oscuros, menor electricidad en la atmósfera. Desnutrición o desequilibrio alimentario; por ejemplo, la glotonería "de ahi su frecuencia en niños que comen sin mesura" "5,21; también comidas mal preparadas o "malos alimentos" como legumbres harinosas, carnes blancas blandas y gelatinosas. Había criterios dispares acerca del consumo de leche, recomendado por algunos y desaconsejado por otros por estimar que favorecía el desarrollo de las escrófulas. Se informaba que casi 100 años antes Boerhaave y después otros habían señalado los efectos perniciosos de la leche de una nodriza escrofulosa o debilitada por enfermedades o excesos, que alteraban la calidad del líquido ${ }^{16}$.

Otras causas mencionadas eran: sedentarismo o ejercicio violento, abuso del sueño o vigilias excesivas, trabajos intelectuales, pasiones del alma, celibato, abuso del coito, masturbación. Baños tibios, embarazo, corte de pelo frecuente en los niños. Enfermedades anteriores como sarampión, viruela, sífilis, escorbuto, fiebre reumática $\mathrm{u}$ otras afecciones debilitantes. A veces se atribuía a consecuencia de tratamientos, por ejemplo, el efecto pro-escrofuloso del sublimado corrosivo en la sífilis. Uso inmoderado de narcóticos, eméticos, purgantes o sangrías. Se indicaba que a menudo la escrofulosis era desencadenada por golpes, torceduras, magulladuras $\mathrm{u}$ otros accidentes ${ }^{4}$.

Dos temas que seguían siendo polémicos en el siglo
XIX respecto a la causa de la escrofulosis eran el papel de la herencia y del contagio por medio de un agente morbífico especial desconocido o "virus". Había defensores y detractores del carácter hereditario de la enfermedad ${ }^{22}$. Sin embargo, tendía a imponerse la hipótesis de la herencia como causa solamente de la constitución escrofulosa. Los autores que descartaban el contagio aducían que juntando niños escrofulosos y sanos hospitalizados en las mismas salas, éstos no adquirían la enfermedad. Se citaban experimentos infructuosos de médicos que trataron de transmitir la enfermedad mediante masaje o inoculación de materia escrofulosa a niños sanos y animales ${ }^{16,17}$.

\section{Patogenia}

La mayoría de las propuestas acerca de la patogenia de la escrofulosis se insertaban en el marco de la teoría humoral hipocrática, con modificaciones matizadas por los conceptos imperantes en cada época o región. Así, se atribuyó a la viscosidad grasosa de la flema al combinarse con bilis negra, a una flema pútrida y salada, y a una deficiente eliminación de ácidos corporales ${ }^{23}$. Se sugería que una linfa espesada se depositaba en vasos y tejido ganglionar. Tal espesamiento podía originarse del efecto de un ácido, estancamiento de la linfa por alteración de la relajación y dilatación de los vasos linfáticos, presencia de líquido seminal en la linfa o por un aumento de la actividad absorbente del ganglio. Se mencionaba también la influencia de una constitución en que predominara el desarrollo y dinamismo del sistema linfático sobre el sanguíneo, lo que conducía a una mayor actividad de los ganglios. Esto precisamente ocurriría en individuos con temperamento linfático o flemático, en quienes el efecto de causas irritantes se manifestaría por la hinchazón de las partes afectadas y lesión de otros órganos como el pulmón, hígado y grupos ganglionares alejados, que simpatizarían con el ya afectado ${ }^{4}$.

\section{Patología}

La caracterización de la lesión ganglionar se limitaba a observaciones clínicas y macroscópicas y se empleaban términos genéricos, como tumor escrofuloso. Las lesiones caseosas de los ganglios podían ser de tamaño variable, a veces apenas visibles, otras del tamaño de una arveja o mayores, extendiéndose a veces a todo el ganglio ${ }^{24}$. Se describían también como tubérculos, "infiltración tubercular" o "tuberculización amarilla" ${ }^{10,25}$. Como se ha mencionado, hacia mediados del siglo XIX ya se hablaba de la adenitis cervical escrofulosa o estrumosa, que podía tener una marcha aguda o crónica ${ }^{26}$. Algunos consideraban que no todos los tumores escrofulosos eran 
adenitis; también destacaban que no era fácil distinguir la escrófula de la degeneración cancerosa ${ }^{27}$.

En cuanto a diferenciar la escrófula de la tuberculosis, había quienes sostenían que mientras no se probara que existían constantemente tubérculos en los ganglios linfáticos, huesos y otros órganos afectados, los males escrofulosos permanecerían como enfermedades aparte y distintas de los tubérculos ${ }^{28}$.

\section{Pronóstico}

Considerando el espectro de las enfermedades llamadas escrofulosas, Lepelletier las agrupaba de acuerdo con su evolución más o menos favorable. Así reputaba como las más graves la tisis tuberculosa y la tumefacción de los ganglios mesentéricos, ambas frecuentemente mortales. Le seguían en gravedad: la "vertebralitis", la luxación espontánea, y el tumor blanco o artritis fungiforme de los niños. Las dos primeras no tenían tratamiento efectivo; la última era susceptible de ser curada por amputación. En tercera categoría agrupaba las hidropesías escrofulosas, las hidrartrosis escrofulosas, el broncocele escrofuloso (bocio) y los abscesos escrofulosos profundos, generalmente menos aflictivos que los del grupo anterior, aunque podían llegar a ser mortales si se trataban inadecuadamente. En una última categoría colocaba las tumefacciones y supuraciones de los ganglios linfáticos exteriores, los catarros escrofulosos y las úlceras cutáneas escrofulosas: siendo mucho menos graves que las anteriores, solían ser muy rebeldes y su curación se lograba después de mucho tiempo con un manejo racional. Estas generalmente no llevaban a la muerte, a menos que se las descuidara o que el enfermo cayera en manos de charlatanes ${ }^{16}$.

\section{Comentario}

En el siglo XIX el prototipo de la enfermedad escrofulosa continuó siendo el compromiso de los ganglios linfáticos cervicales, catalogado de adenitis por algunos, que se presentaba especialmente en la niñez y juventud. Su alta frecuencia se apreciaba por la palpación o la apreciación de cicatrices. Se estimaba que era un mal que podía curarse, pese a su evolución arrastrada en algunos casos.

Al considerarse la escrófula asociada a un tipo determinado de constitución del individuo, y al aceptarse que también podían afectarse otros órganos o sectores corporales, algunos la catalogaban como una "enfermedad constitucional". Tal designación no implicaba una idea unívoca. Algunos la concebían solo como una enfermedad diseminada en todo el cuerpo, a diferencia de las enfermedades tópicas que se limitaban a un solo punto. Otros pensaban que, a diferencia de una "enfermedad general", que obligaba a los enfermos a guardar cama, la constitucional les posibilitaba desempeñar ciertas actividades ${ }^{29}$. No obstante, la idea más aceptada de enfermedad constitucional señalaba que era una afección crónica que parecía inherente a la constitución y que después de atacar un órgano terminaba por afectar todos los sistemas órgánicos, es decir, la constitución entera ${ }^{30}$.

Alternativamente se clasificaba también la escrófula como "enfermedad diatésica". Para muchos este nombre era equivalente a enfermedad constitucional. Otros afirmaban que las enfermedades diatésicas tenían caracteres propios, entre los cuales se mencionaban los siguientes: formar determinado producto morboso que podía afectar todos los sistemas orgánicos, como el tubérculo y el cáncer. Disminuir la resistencia del organismo, predisponiéndolo a determinadas dolencias e imprimiendo a sus reacciones una fisonomía especial: esto ocurriría en la escrófula, el artritismo y el herpetismo. Impregnar la totalidad del organismo transformando la vida del individuo a una "vida diatésica" ${ }^{31}$. Facilitar el desarrollo y progreso de la enfermedad aun después que hubiera cesado de actuar la causa accidental que la había producido ${ }^{29}$.

Los escritos sobre las causas de la tuberculosis pulmonar en Francia y Gran Bretaña en el siglo XVIII consideraban una constelación de factores. Entre ellos se han identificado cuatro modelos principales: contagio, alteraciones fisiológicas, predisposiciones hereditarias y estilos de vida. La idea de contagio de la tisis dominó en los países del sur de Europa en los siglos XVII y XVIII, donde se prescribieron medidas para controlar y prevenir la enfermedad. La teoría de una "disposición" hereditaria avanzó en el XVIII para predominar en el XIX con la denominación de "diátesis", sobre la cual actuarían los factores ambientales y de estilo de vida ${ }^{32}$. Las propuestas etiológicas que aquí se han enumerado para desencadenar la escrófula en sujetos predispuestos incluyen principalmente factores ambientales, y otros correspondientes a costumbres o estilo de vida.

En la segunda mitad del siglo XIX se desarrollaba la ciencia de la microbiología. La etiología bacteriana y la noción de contagio pasarían a ocupar un lugar destacado en la medicina del siglo XX, modificando el conocimiento de la etiología y patogenia de la escrófula y la tuberculosis. Se eliminaría progresivamente la consideración diatésica de enfermedades como escrófula, tuberculosis, escorbuto, gota y sífilis ${ }^{33}$.

\section{Resumen}

En el siglo XIX la escrófula era frecuente, estimada por la percepción de nódulos o cicatrices en el cuello. Paulatinamente se le empezó a denominar adenitis escrofulosa. Podía presentarse en personas de ambos sexos en 
todas las épocas de la vida; pero era más frecuente en la infancia. Se pensaba que afectaba principalmente a sujetos con una constitución flemática heredada que implicaba una susceptibilidad o diátesis escrofulosa. La enfermedad sería desencadenada por agentes ambientales, hábitos, o excesos en el estilo de vida. Aparte de los ganglios linfáticos cervicales, podía afectar otros grupos ganglionares, huesos, articulaciones, pulmones y otras vísceras, atribuyéndosele diversas formas de presentación que variaban entre las potencialmente curables a las frecuentemente mortales. La afectación multiorgánica, la caseificación y la "tuberculización" de las lesiones originaron la discusión sobre si la escrófula y la tuberculosis eran una sola enfermedad o dos diferentes, y si se desencadenaban sobre una diátesis común o cada una sobre una diátesis específica escrofulosa o tuberculosa. En la mayor parte del siglo XIX , antes del descubrimiento del bacilo de Koch, la noción de contagio como causa de la escrófula y de la tuberculosis pulmonar no parecía predominar en países europeos.

\section{Referencias bibliográficas}

1.- Duarte I, Chuaqui C. Historia de la escrófula: de la discrasia humoral a la consunción. Rev Med Chile 2016; 144: 503-7.

2.- Duarte I. La pretendida curación de la escrófula por el toque del rey. Rev Chilena Infectol 2014; 31: 459-67.

3.- Scarano G B. Cenna storici sulla eziopatogenesi della scrofula a meta' dell'800. Riv Stor Med 1969; 13: 210-3.

4.- Diccionario de Ciencias Médicas. Traducción del francés al castellano. Tomo XIII. Madrid: Imprenta de Don Mateo Repullés; 1823. Escrófulas; p. 52-93.

5.- Gerdy N. Pathologie génerale médico-chirurgicale. Paris: Victor Masson; 1851. p. 93-94.

6.- Lebert $\mathrm{H}$. Traité pratique des maladies scrofuloses et tuberculoses. Paris: J. B. Baillière; 1849. 811 p.

7.- Fabre $\mathrm{F}$ A. Bibliothèque du médecin-praticien. Tomo 6. Maladies des enfants, de la naissance a la puberté. Paris: JB Baillière; 1847. p. 557-616.

8.- Villemin J A. Études sur la tuberculose: preuves rationnelles et expérimentales de sa spécifité et de son inoculabilité. JB Ballière et fils. Paris 1968. Reimpresión: Breignisville, Pennsylvania, USA: Nabu Public Domain Reprints; 2015. p. 262.

9.- Ancell H. A treatise on tuberculosis, the constitutional origin of consumption and scrofula. Longman, Brown, Green and Longmans: Londres; $1852.779 \mathrm{p}$.

10.- Copland J. A dictionary of practical medicine. Vol III, part I. London: Longman, Brown, Green Longmans \& Roberts; 1858. Scrofula and tubercles; p. 731-68.

11.- Kent J. Observations on the cause, symptoms, and nature of scrofula or king's evil, scurvy and cancer. 8a edición, Bury St Edmonds: J. B. Frost; 1833. 42 p.

12.- Bouchut E. Nouveaux éléments de Pathologie Générale et de Séméiologie. $2^{\mathrm{a}}$ Edición. París J.B. Baillière et fils; 1869. p. 244-245.

13.- Da Costa J C. Modern Surgery, $4^{\text {th }}$ Edition, 1903. Capítulo
13. Tuberculosis and scrofula; p. 177-186. Disponible en: http://jdc.jefferson.edu/dacosta_modernsurgery/46. (Consultado: 28 de junio de 2016).

14.- Arnaud-Lesot. Aux XIX ${ }^{\mathrm{e}}$ siècle, les beaux et les laids scrofuleux. Rev Prat 2003; 53: 1735-7.

15.- Schapter T. Scrofula. En: Tweedie A, Editor. A system of practical medicine. Philadelphia: Lea and Blanchard; 1841. p. 238-76.

16.- Lepelletier A. Traité complet sur la maladie scrophuleuse et les differentes varietés qu'elle peut offrir. París: Germe Ballière; $1830.326 \mathrm{p}$.

17.- Phillips B. Scrofula: its nature, its causes, its prevalence, and the principles of treatment. Philadelphia: Lea and Blanchard; 1846. Reimpreso por General Books LLC, Memphis, USA, 2012. $129 \mathrm{p}$.

18.- Hardy F, Lefeuvre J. Lecciones sobre la escrófula y las escrofulides y las sifilides. Madrid: Imprenta Española; 1866. p. 30.

19.- White T. A treatise on struma or scrofula, common called the king's evil. Londres: J. Murray; 1784. 110 p.

20.- Dubos R, Dubos J. The white plague: tuberculosis, man and society. New Brunswick, New Jersey: Rutgers University Press; 1996. p. 9-10.

21.- Landoucy L. Le toucher des écrouelles. L'Hôpital Saint Marcoul. La maladie du Roi. París: Masson; 1907. 40 p.

22.- Lomax E. Hereditary or acquired disease? Early nineteenth century debates on the cause of infantile scrofula and tuberculosis. J Hist Med Allied Sci 1977; 32: 356-74.

23.- Carmichael R. An essay of the nature of scrofula with evidence of its origin from disorder of the digestive organs. London: John Callow; 1810. p. 26.

24.- Wagner E. A manual of General Pathology. For the use of students and practitioners of Medicine. New York: William Wood \& Company; 1876. p. 444-5.

25.- Druitt $R$. The principles and practice of modern surgery. Philadelphia : Henry C Lea; 1867. Chapter 13, Scrofula and tuberculosis in general; p. 104-9.

26.- Monneret E, Fleury L. Tratado completo de Patología Interna. Tomo IV, De la degeneración tuberculosa de los ganglios y de las escrófulas en general. Barcelona: Viuda de Jordán e Hijos; 1845. p 77-92.

27.- Denonvilliers C, Gosselin L. Compendium de Chirurgie Pratique. Tomo II. París: Labe; 1851. p 184.

28.- De la Berge L, Monneret E, Fleury L. Compendium de médecine pratique. París: Bechet Jeune; 1846. Scrofules ou Scrophules; p. 439-44.

29.- Folch F P. Tratado elemental de Patología General y Anatomía Patológica. Barcelona. Imprenta y Librería de Benito Espona, 1843. p. 52-53.

30.- Diccionario de medicina. Tomo I. p 481. París: Librería de Rosa y Bouret; 1854. Constitucional; p. 481.

31.- Ribera J. Estudio de las relaciones recíprocas entre los estados morbosos generales y las lesiones quirúgicas. Memoria Premiada en el Concurso de 1886. En: Anales de la Real Academia de Medicina. Tomo VII, Cuaderno primero. Madrid, Imprenta del Cosano, 1943. p. 40-1.

32.- Bernier J. L'interprétation de la phtisie pulmonaire au XVIII ${ }^{\mathrm{e}}$ siècle. CBMH/BCHM, 2005; 22: 35-56.

33.- Ackerknecht EH. Diathesis: the word and the concept in medical history. Bull Hist Med, 1982; 56: 3117-25. 